\title{
A Start-Timing Detector for the Collider Experiment PHENIX at RHIC-BNL
}

\author{
K. Ikematsu, Y. Iwata, K. Kaimi ${ }^{\ddagger}$, M. Kaneta, T. Kohama, \\ N. Maeda ${ }^{a}$, K. Matsukado, H. Ohnishi, K. Ono, A. Sakaguchi ${ }^{b}$, \\ T. Sugitate, Y. Sumi, Y. Takata, M. Tanabe ${ }^{c}$ and A. Yokoro \\ Department of Physics, Hiroshima University, \\ Kagamiyama, Higashi-Hiroshima 739-8526, Japan
}

\begin{abstract}
We describe a start-timing detector for the PHENIX experiment at the relativistic heavy-ion collider RHIC. The role of the detector is to detect a nuclear collision, provide precise time information with an accuracy of 50ps, and determine the collision point along the beam direction with a resolution of a few $\mathrm{cm}$. Technical challenges are that the detector must be operational in a wide particle-multiplicity range in a high radiation environment and a strong magnetic field. We present the performance of the prototype and discuss the final design of the detector.

PACS number: 29.40.Ka
\end{abstract}




\section{Introduction}

The PHENIX (Pioneering High Energy Nuclear and Ion eXperiment) spectrometer [1] of the Relativistic Heavy Ion Collider (hereafter abbreviated as RHIC) at the Brookhaven National Laboratory is now under construction. Phenix is composed of various sub-detectors for hadron-, lepton- and photon-measurements in ultrarelativistic heavy nucleus-nucleus collisions at $\sqrt{s}=200 \mathrm{GeV} / c$ per nucleon, as well as in proton on proton reactions at $\sqrt{s}=500 \mathrm{GeV} / c$. For particle identification of interesting species and background rejection, time-of-flight (hereafter abbreviated as TOF) measurements are the crucial function. The RHIC beam bunches have a large longitudinal dimension, which is expected to be about $25 \mathrm{~cm}$ rms in the present accelerator design. It turns out that a nuclear collision could happen at any place in the colliding region and at any moment during the beam bunch crossing, which continues for about 2ns. For precise TOF measurements with detectors providing stop timing signals with 100ps resolution, we have to determine the start time very accurately. A detector sub-system to measure the start timing is named Beam/Beam Counter (abbreviated as BBC hereafter) in PHENIX.

A pair of $\mathrm{BBC}$ will detect secondary particles from every collision at very forward angles between $2.4^{\circ}$ and $5.7^{\circ}$ in the north- and south-ends of the central spectrometer along the beam axis. Each measures the arrival time of a leading charged particle, which travels at nearly the speed of light. An average and a difference between time measured at each end provide the start time measured from a common clock and the longitudinal vertex position along the beam axis, respectively. The vertex position will be employed in the first level trigger to clean up events and as a starting point for further online tracking analysis.

We have completed design studies of the BBC consisting of an array of 64 identical detector elements at each end. We have tested prototype detector elements with pion beams, studied performance of a small array of the prototypes in heavy-ion collisions at $15 \mathrm{GeV} / c$ per nucleon, and have recently carried out quality assurance tests with the detector elements of the production phase.

In section 2, we describe requirements, conceptual design and the technological choice for the detector. Results from a series of beam tests with the prototypes are

given in section 3 . In section 4 , we discuss on our technological choice and optimization. We also demonstrate the start time determination with multiple measurements of reaction products from nuclear collisions and show the results from the QA tests. A summary is given in section 5 .

\section{Design of Detector Element and Array}

Fig. 1 shows a cut-away view of the PHENIX spectrometer. The BBC is positioned between the central spectrometer magnet and the muon sub-detector, and covers a pseudo-rapidity region from 3.0 to 3.9 over full azimuth. Another identical set is installed in the opposite side. Secondary particles emitted in this kinematical region pass through an axial hole of the main spectrometer magnet and go into the BBC. 
All the strongly interacting particles will be finally absorbed in the piston magnet placed immediately behind the BBC.

The BBC must satisfy the following requirements: It has to primarily provide a precise time information of each collision in various environments of particle multiplicity. In case of $\mathrm{p}+\mathrm{p}$ reactions, one would expect only a few charged particles in this acceptance. For a nuclear collision such as a central $\mathrm{Au}+\mathrm{Au}$ collision, however, one would expect more than 1,000 charged particles hitting the BBC. The detector has to be segmented to possess capability to handle the wide dynamic range of particle multiplicity as the entire system, and each detector element has to be well designed for multi-particle hits.

Since the BBC will be placed at a very forward angle, it will be irradiated with an enormous amount of charged and neutral particles from beam-beam collisions, albedo particles from materials surrounding the detector, and more seriously with beam associated backgrounds. A simulation estimates that the gamma- and neutronflux at the position might be about $10^{10}$ and $10^{11} / \mathrm{cm}^{2} /$ RHIC-year, respectively. The detector must be resistant against such a large radiation dose. In addition to this, due to geometrical restrictions of the PHENIX configuration, access to the BBC after the rolling in is very limited. The detector has accordingly to work maintenance free for a long period.

There is a magnetic field of about $0.3 \mathrm{~T}$ mostly parallel to the beam axis under the normal magnet operation. The detector must operate under such a high magnetic field, since the field is too strong to shield with any magnetic materials down to an operational level of standard photomultiplier tubes.

Our technological choice is to build the BBC as an array detector composed by 64 identical Cherenkov counters. Figs $2 \mathrm{a}$ and $2 \mathrm{~b}$ depict the individual detector element and the array mounted on a mechanical frame. The detector element consists of four components; a Cherenkov radiation material, a photomultiplier tube, a high-voltage divider module and mechanical accessories to pack and mount them on the frame. The Cherenkov radiator and the photomultiplier tube are made to be in one body, in order to avoid using any glue or optical grease to join the two parts together. The Cherenkov radiator component, which is in fact a very thick window of the photomultiplier tube, is made of fused Quartz and has a hexagonal shape inscribing 1" diameter circle and $30 \mathrm{~mm}$ long. The photomultiplier tube was designed on the basis of an existing tube of 1" in diameter, Hamamatsu R3432, which has 15 layers of fine mesh dynode and is supposed to be operational in a magnetic field. Several prototypes of photomultiplier elements were produced in Hamamatsu Photonics and tested with pion beams at KEK and with heavy ion beams at AGS-BNL.

\section{Results from Prototype Tests}

\subsection{Prototype tests with pion beams}

Three rounds (T285, T298 and T322) of beam tests were carried out at the $12 \mathrm{GeV}$ Proton Synchrotron facility of KEK. A typical setup at the beam line T2 is illustrated 
in Fig. 3. We used a negative pion beam with its momentum of $1.6 \mathrm{GeV} / c$. A typical beam rate was around $1,000 / \mathrm{cm}^{2}$ in a $1.6 \mathrm{~s}$ beam spill with a beam definition of;

$$
D E F 1 \otimes D E F 2 \otimes D E F 3 \otimes D E F 4 \otimes R E F 1 \otimes R E F 2,
$$

which was used as a trigger for data taking. A 35cm-long solenoid magnet with an inner bore radius of $10 \mathrm{~cm}$ was employed to generate a magnetic field. The prototype detector element was placed at the center, where the magnet produces almost a flat field within $0.2 \% / \mathrm{cm}$ along the axis.

ADC and TDC data from the prototype detector labeled TEST and two time reference counters, REF1 and REF2, were read out with standard CAMAC modules by a PC based DAQ system. The ADC spectrum of the prototype detector and the TOF spectrum between the prototype and one of reference counters are shown in Figs. $4 \mathrm{a}$ and $4 \mathrm{~b}$, respectively. The ADC spectrum shows a single peak, however, that can not be approximated by either a Poisson or a Gaussian distribution.

The intrinsic timing resolution of 100ps is achieved under the magnetic field of 0.3T by using a leading edge discriminator without any software cuts or corrections. It is improved up to 50ps with the standard slewing correction [2] using the ADC information without any cuts. The typical TOF spectrum after the correction is seen in Fig. 4c. Dependence of the current gain and the intrinsic timing resolution were studied as a function of strength of applied magnetic field in Fig. 5. There is a clear drop of ADC gain by 30\% immediately after the magnetic field was switched on, but shows a plateau until $0.3 \mathrm{~T}$ and again decreases gradually beyond this field strength. The timing resolution after corrections, however, does not indicate any degradation and keeps the performance at the same level.

\subsection{Prototype test with heavy-ion beam}

The performance of the prototype detector element for single particles was studied using pion beams, but in reality we also need to examine characteristics of the performances for a multi-particle shot in a detector array. For this purpose, another test was carried out using heavy-ion beams from the AGS at BNL. The test setup in Fig. 6 was placed downstream of the MPS experiment on the A1 line, and measurements were performed with a gold beam at $14.5 \mathrm{GeV} / c$ per nucleon impinging on a $5 \mathrm{~mm}$ thick lead target. The beam envelope was defined by a beam defining counter BDF. Two time reference counters, REF1 and REF2 were placed in front of the target. They provide the start time signals for TOF measurement and measure the charge of the incident heavy ion. A nuclear reaction was detected with a small pulse height in a beam-veto counter VETO and with large pulse heights in tagging counters, TAG1 and TAG2, which were placed in front of the test detectors. Seven prototype detector elements were assembled to form an array with the realistic gap spacing. ADC and TDC data from all the counters were read out with standard CAMAC modules by a PC based DAQ system. Data were accumulated with a trigger of;

$$
B D F \otimes R E F 1 \otimes R E F 2 \otimes T A G 1 \otimes T A G 2 \otimes \overline{V E T O} \otimes\left[\sum_{i=1}^{7} T E S T_{i}\right] .
$$


The array was set at $15^{\circ}$ with respect to the beam line, and the distance from the target to the detectors was typically $35 \mathrm{~cm}$. We could expect around 10 charged particles entering the array from a central collision.

Fig. 7a shows an ADC distribution of one of the prototype detector positioned at the center of the array, and Fig. 7b shows the TOF spectrum between the detector and one of the time reference counters. The ADC spectrum is quite different from those of the pion beam tests in Fig. 4a. It implies a large amount of background particles hitting the detector with small pulse heights and they might hide the ADC peak for a single particle hit. It was found that the majority of background particles came from adjacent detectors. The same slewing correction process applied to the pion beam data, was employed to this data. The timing resolution of 54ps was achieved in Fig. $7 \mathrm{c}$, but the resolution before the correction is worse than that of pion beam tests, because of the much broader ADC spectrum.

\section{Discussion}

\subsection{Detector concept}

In order to accept leading particles traveling at the speed of light, the BBC must be set in a small space at the very forward angle. Here the particle multiplicity per unit area could be extremely high for central nuclear collisions. The multiplicity, however, varies greatly depending on the impact parameter of the collision in question. Consequently, the number of particles hitting individual detector elements varies, and the detector elements must accommodate multiple particles entering anywhere on the face of the detector.

Once we consider a possibility of detector coupled with a photon counter, it is a natural solution to employ Cherenkov radiation light instead of scintillation light. A fast charged particle passing through a transparent material will radiate Cherenkov light. A photon counter optically coupled to this material will measures the light. Such Cherenkov light is always produced, but is usually negligibly small compared to the light signal from a scintillating material. However, if multiple particles enter a single detector, the Cherenkov light becomes large enough to fire a discriminator and trigger a TDC a few hundred ps prior to the scintillation light, which comes later due to the larger time constant of the scintillating process. This timing gap prevents precise timing measurements.

Another difficulty of precise time measurements in high multiplicity environments is that the photon counter should be placed at a position where the photon counter can view the entire detection volume at a constant distance. Otherwise the photon counter measures the earliest time from light generated in the nearest hit spot. We would then see considerable time spread originating from variation of the optical path length inside the detector. In other words, a detector designed like a paddle counter even viewed with double photon counters at the both ends would not work well in this case.

The solution that we chose is a compact Cherenkov detector with a high-speed 
photomultiplier tube immediately behind the radiator material along the particle direction, and multiple detector elements form an array surrounding a beam pipe at very forward angles.

\subsection{Radiator shape optimization}

The Cherenkov radiator shape must be optimized to collect the maximum number of photons. We have two parameters to be determined; the radiator length determining the number of photons produced, and the outer shape determining the photon collection by internal reflection onto the cathode plane. The speed of light in a Quartz radiator is slower than the speed of light in vacuum by a factor of the index of refraction. The path length of photons traversing the radiator is much longer than that of the particle, since the photons are radiated at the Cherenkov angle of about $45^{\circ}$ in Quartz with respect to the direction of the particle. As the result, Cherenkov photons produced near the front of radiator arrive at the photo-cathode later than the particle, whereas the arrival time of photons produced at the back of radiator is almost the same as that of the particle. This fact introduces a spread $\left(\sigma_{1}\right)$ of photon arrival time and the spread results in deterioration of timing resolution of the detector. To minimize the spread, the length of the radiator must be as short as possible. On the other hand, a reasonable number of photons in a photomultiplier tube is necessary for a good signal ratio to noise and for reduction of another time spread $\left(\sigma_{2}\right)$, which is originated from fluctuation of traveling time by photo-electrons from the cathode to the anodes in a photomultiplier tube. A larger number of photo-electrons reduces the fluctuation smaller. This temporal fluctuation is characterized by the transit time spread for a single photon detection, which is measured to be $0.33 n$ for this particular photomultiplier tube.

In Fig. 8, we show the results of a simple model calculation with an assumption of the photo-cathode sensitivity $N_{0}$ of 100, for several values of the mixing parameter

$c$, where $\sigma=\sqrt{c \times \sigma_{1}^{2}+\sigma_{2}^{2}}$. If we take $c=1$, the optimized radiator length is around $15 \mathrm{~mm}$. In order to verify the calculation and to study the effects of outer shape of radiator, we have tested several prototype counters with hexagonal and circular shaped radiators of different lengths. In Fig. 9, we plotted the test results of the timing resolution as a function of the radiator length. The model calculation with the parameter $c \approx 0.25$ seems to explain the global tendency rather well. Though the model is too simple to explain everything, we could understand the trend and choose the length to be $3 \mathrm{~cm}$. The shortest possible radiator is used, because a shorter radiator prevents cross talk between adjacent detector elements when a particle enters with a shallow angle.

The ADC spectrum taken with a circular shaped radiator is shown in Fig. 10. Comparing to Fig. 4a, which was taken with a hexagonal radiator, there is a clear difference in the distribution. A Monte Carlo study simulating internal reflection of photons inside the Quartz radiator block explains the dependence of the spectrum shape on radiator shape and length very well. It is because the cathode sensitive area is smaller than the radiator cross section, and some of the photons can not reach to the sensitive area by internal reflection. The probability of this loss is a function of 
the radial position of the incident particle, and the function strongly depends on the radiator shape. We therefore see pulse height dependence on radial hit position in the ADC spectrum. There is no noticeable difference on the timing resolution between the circular and hexagonal radiators. We have chosen the hexagonal shape, because the higher hermeticity is important for proton on proton collisions and because of the preferable single peak feature in the pulse height distribution.

\subsection{Radiation hardness}

Radiation damage in a photomultiplier tube usually results in a gradual lowering of transparency of the window material due to color center creation. Negligibly small effects on the photo-cathode or dynode materials are expected because of their quite low thickness [3]. In this design, the photomultiplier tube has a very thick window compared to a standard tube and high transparencies at short wavelengths are essential to collect the Cherenkov light. The optical degradation of the window material from radiation damage is seriously examined. As described in the previous section, since this thick window serves as a Cherenkov radiator, there is no glue or grease used. This ensures no loss of photons at any joint gap and highly improves the reliability of the detector element both mechanically and in radiation hardness, since glues and grease lose transparency at short wavelength after radiation exposure. We chose fused Quartz of SPRASIL grade as the window material, since it is one of the most radiation hard materials and provides excellent transparency at short wavelength. It has been studied [3] that, for wavelengths down to around 220nm, the Quartz does not indicate any serious degradation of transparency for thermal neutron radiation up to $10^{14} / \mathrm{cm}^{2}$, which corresponds to the total amount of neutron radiation during 1000-year operation at RHIC. We tested radiation hardness of the prototype detector against $\gamma$-rays. The detector was irradiated with $1 \mathrm{MR}$ of $\gamma$-rays from ${ }^{60} \mathrm{Co}$ at the Radioisotope Center of Hiroshima University. The total amount of $\gamma$-ray irradiation is approximately $10^{5}$ times larger than that expected in 1-year RHIC operation, and no deterioration of the detector was observed.

\subsection{Collision time determination}

The purpose of the $\mathrm{BBC}$ is to determine a unique value of the collision time from multiple measurements of secondary particles. This function is demonstrated with a small array of prototype detectors in the heavy-ion beam test. There are two simple ideas to derive the collision time. One is to adopt the smallest TDC value in each event and neglect any other signals. It corresponds to looking at the fastest particle in the collision of interest. Another is to take an average over all the time information in each event. This may include undesirable particles at the velocities slower than the speed of light. These two simple methods are demonstrated in Fig. $11 \mathrm{a}$ and 11b, where TOF spectra between the BBC and a time reference counter REF1 are plotted. For comparison, Fig. 11c shows a TOF spectrum of all the time information available over all events in question. Both of the methods provide the time resolution of around 40ps, which is slightly better than the timing resolution of 
each detector element. Although the number of sample measurements is only seven, it is because of multiple measurements of the single value in each event. In the real experiment, a larger number of independent measurements are expected and a better timing resolution can be obtained accordingly.

\subsection{Quality assurance test}

The quality assurance test (T395) using the real detector elements built in the firstproduction-year was carried out at the $12 \mathrm{GeV}$ Proton Synchrotron facility of KEK. The setup at the beam line T2 is almost the same as that in fig. 3. Fig. 12 shows results of photomultiplier current gain and timing resolution of all the 37 detector elements arranged in order of the gain. The data were taken by applying a constant high-voltage value of $2.3 \mathrm{kV}$ to each photomultiplier tube under a magnetic field of 0.3T. Although the spread of current gain between the lowest- and highest-gain tubes at this voltage extends over a factor of 4 , very stable operation of all detector elements was observed. The timing performance is better than with the prototype detectors, demonstrating the uniformity of the detector production process.

\section{Conclusion}

We have designed a start-timing detector for the PHENIX experiment at RHIC-BNL. The detector is composed of 64 identical detector elements, placed at very forward angles in the opposite ends of the central spectrometer. The detector element is a Cherenkov counter consisting of a $3 \mathrm{~cm}$ long hexagonal shaped Quartz radiator and a photomultiplier tube of 1 " in diameter with 12 stage mesh dynodes. The radiator and the tube are united into one body.

The performance of prototype detectors was studied with pion beams under magnetic fields and with heavy-ion beams. The intrinsic timing resolution of the prototypes was about 100ps without any corrections, and was about 50ps with standard slewing corrections. The test experiment using heavy-ion beams demonstrates good performance in the collision time determination by an array of seven prototype detectors. The collision time resolution is about 40ps with this small detector array, so a better timing resolution is expected for events with larger particle multiplicity in the entire BBC configuration at the PHENIX experiment.

The quality assurance test was performed with 37 real detector elements built in the first-production-year. The results of timing resolution are very stable, and all of the elements attain a timing resolution of $80-90 \mathrm{ps}$ without any corrections. This $10 \mathrm{ps}$ level fluctuation is due to the difference in current gain of the photomultiplier tubes. After slewing corrections, a resolution as good as 30ps is obtained, independent of the current gain.

It is worth noting that, though the detector was developed for a particular experiment, the detector element, which is now commercially available as R6178 from Hamamatsu Photonics, can be employed in any experimental field requiring a fine 
granularity of 1" in space and an excellent timing resolution better than 50ps under a magnetic field.

\section{Acknowledgements}

This study has been motivated for the PHENIX experiment at BNL-RHIC, and the authors are most grateful to the entire PHENIX collaboration for invaluable discussions during the studies and in particular for the permission to use the picture of Fig.1. The authors also express their sincere thanks to Prof. J. Chiba and the staff of the KEK $12 \mathrm{GeV}$ PS facility for their excellent work and support. They are also indebted to Dr. K. Foley and Dr. E. Asher for their kind offer sharing the heavyion beam at MPS-AGS. Their gratitude is also extended to the MPS technical staffs for their invaluable support. Data analysis was primarily carried out at the DataAnalysis Laboratory for High-Energy Physics, Hiroshima University. This work is supported by a Grant-in-Aid for Scientific Research (05452029) by the Ministry of Education, Science, Sports and Culture, Japan and in part by the U.S.-Japan High Energy Physics Collaboration Treaty.

$\ddagger$ deceased

${ }^{a}$ present address: Physics Department, Florida State University, Tallahassee, FL, USA

${ }^{b}$ present address: Physics Department, Osaka University, Osaka, Japan

${ }^{c}$ present address: Nippon Mikuniya Co., Tokyo, Japan 


\section{References}

[1] S. Nagamiya, Nucl. Phys. A566(1994)287c; Phenix Conceptual Design Report, 29 January 1993, Brookhaven National Laboratory; Phenix CDR Update, November 1993, Brookhaven National Laboratory

[2] T. Sugitate et al., Nucl. Instr. and Meth. A249 (1986) 354; T. Kobayashi and T. Sugitate, Nucl. Instr. and Meth. A287 (1990) 389

[3] The Superconducting Super Collider, Radiation Effects At The SSC, ed. M. Gilchriese, SSC-SR-1035, June 1988; Photomultiplier Tube, April 1993, Hamamatsu Photonics. 


\section{Figure Captions}

Fig.1 A cut-away view of the PHENIX spectrometer at RHIC-BNL. The BBC is depicted surrounding the beam pipe between the central magnet and the muon magnet. There is no access way to the BBC after rolling-in of the spectrometer.

Fig.2 (a) Components of the detector element; a photomultiplier tube of 1" in diameter with a 30mm thick Quartz window, a breeder circuit, an aluminum attachment glued on the top of window, a sleeve of $0.2 \mathrm{~mm}$-thick stainless steel and a plastic sleeve insert. (b) The detector elements mounted on the frame of $30 \mathrm{~cm}$ in diameter and $25 \mathrm{~cm}$ long. The frame can be split into two parts at the middle plane in order to put a 3 " beam pipe in the $10 \mathrm{~cm}$ diameter hole at the center.

Fig.3 A typical setup view of the KEK tests with pion beams. Not in scale.

Fig.4 (a) ADC distribution of a prototype detector with pion beams. TOF spectra between the prototype detector and REF1 (b) before and (c) after the slewing corrections.

Fig.5 The ADC peak channel and the intrinsic timing resolution of a prototype detector with pion beams, as a function of the strength of applied magnetic field.

Fig.6 A setup view of the prototype array tests with heavy-ion beams at AGS. Not in scale.

Fig.7 (a) ADC distribution of a prototype detector positioned at the center of array. TOF spectra between the detector and REF1 (b) before and (c) after slewing corrections.

Fig.8 Timing resolution expected in a model calculation as a function of radiator length.

Fig.9 The test results of intrinsic timing resolution and ADC peak channel as a function of radiator length. Measured with pion beams.

Fig.10 ADC distribution measured by a prototype detector with a circular shape radiator. The distribution is quite different from that of a hexagonal shaped radiator in fig. $4 \mathrm{a}$.

Fig.11 Demonstration of the collision time determination in heavy-ion collisions with an array of seven prototype detectors. (a) TOF spectrum between REF1 and a detector measured the smallest TDC value in each event. (b) TOF spectrum between REF1 and an average of all the valid TDC values measured in each event. (C) TOF spectrum between REF1 and all the detectors measured valid TDC values over the events. The top two spectra contain $13 \mathrm{k}$ events and the bottom one contains $58 \mathrm{k}$ hits. The average hit-multiplicity is 4.6 . They show the timing resolution of $43 \mathrm{ps}, 37 \mathrm{ps}$ and $48 \mathrm{ps}$, respectively. 
Fig.12 The results of quality assurance test performed with pion beams at KEK. 37 detector elements constructed in the first-year-production-phase are tested for the timing resolution and the current gain. 
This figure "figure01.gif" is available in "gif" format from: http://arxiv.org/ps/physics/9802024v1 
This figure "figure02a.gif" is available in "gif" format from: http://arxiv.org/ps/physics/9802024v1 
This figure "figure02b.gif" is available in "gif" format from: http://arxiv.org/ps/physics/9802024v1 
This figure "figure03.gif" is available in "gif" format from: http://arxiv.org/ps/physics/9802024v1 

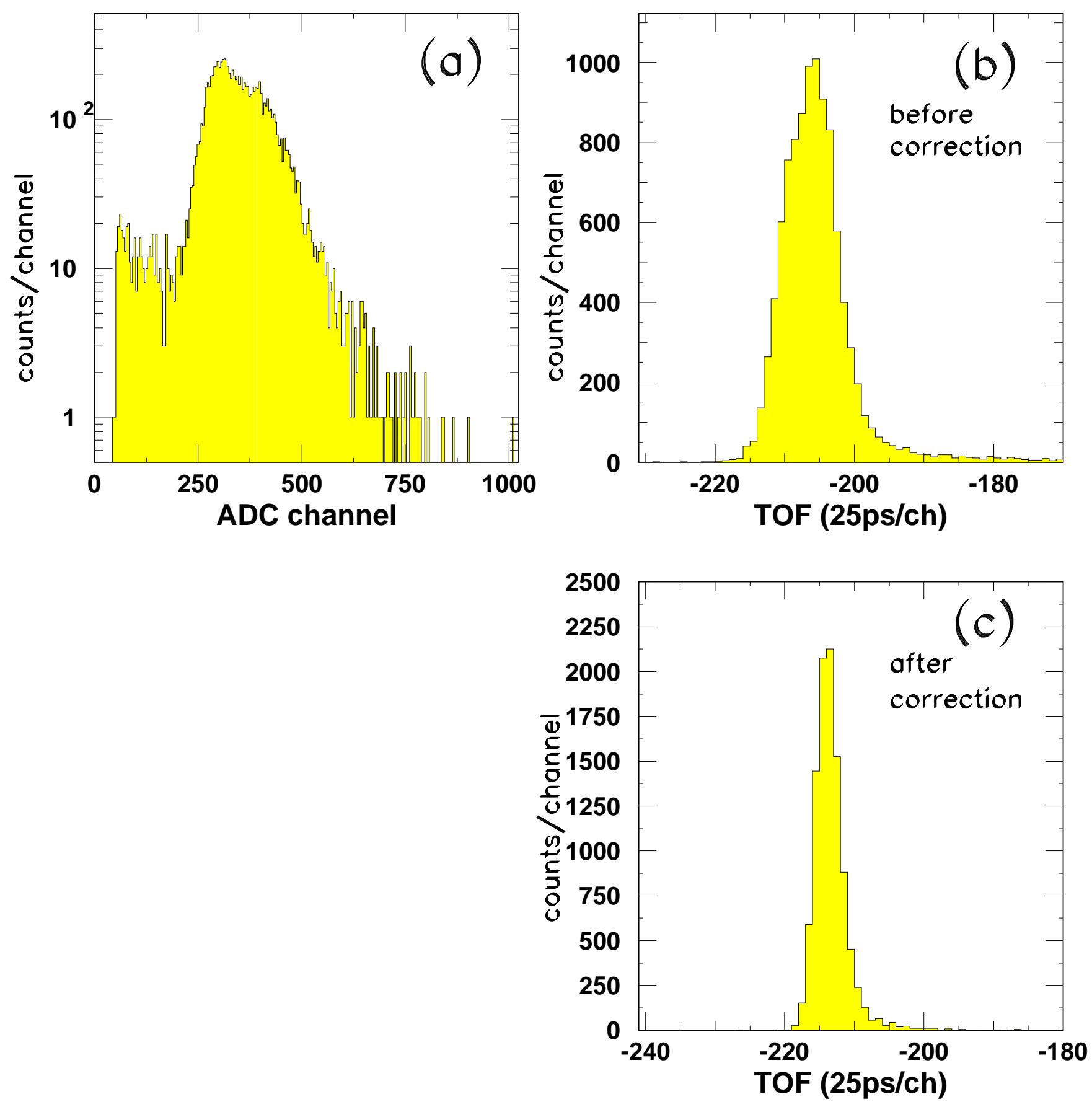
This figure "figure05.gif" is available in "gif" format from: http://arxiv.org/ps/physics/9802024v1 
This figure "figure06.gif" is available in "gif" format from: http://arxiv.org/ps/physics/9802024v1 

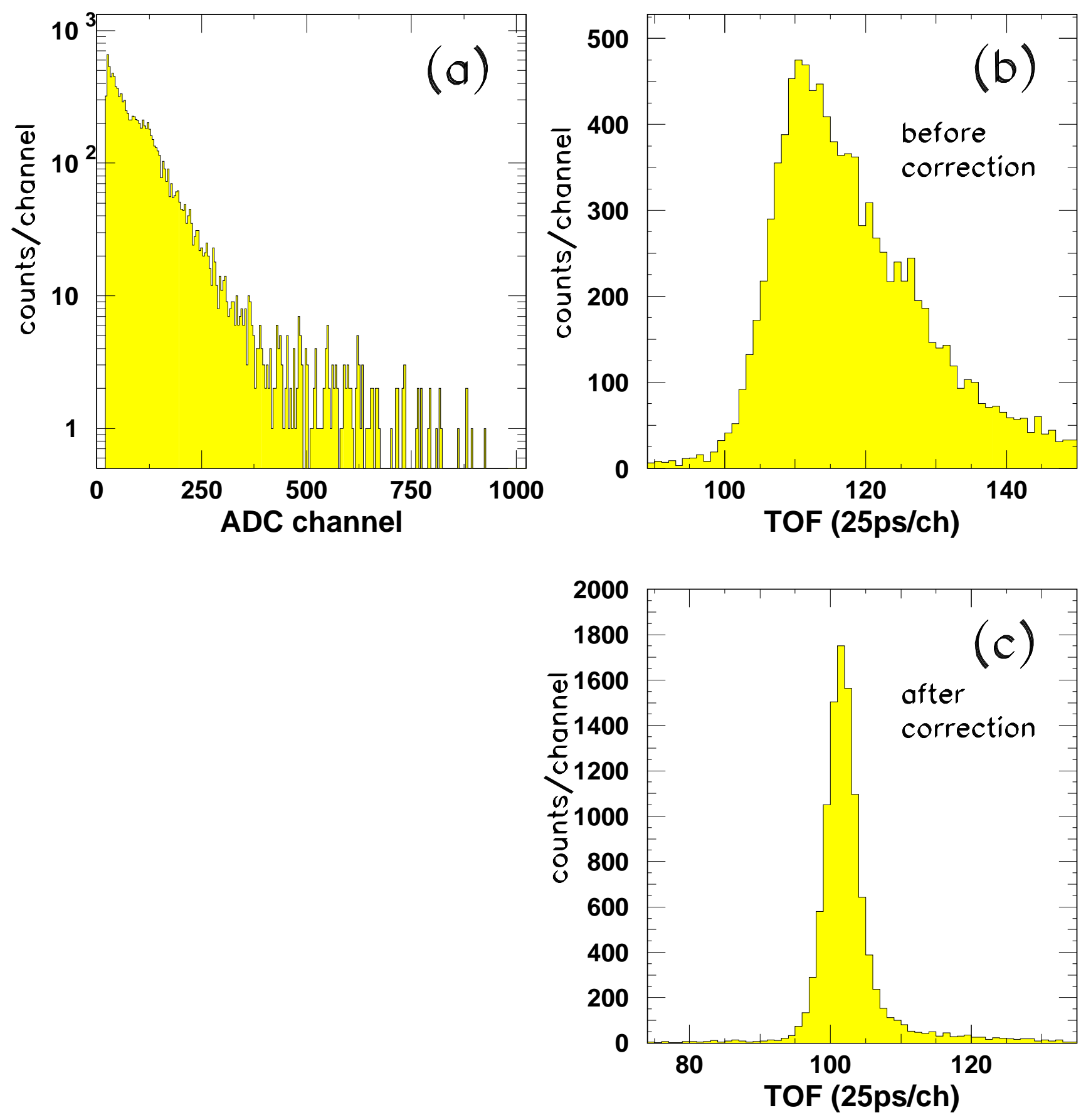
This figure "figure08.gif" is available in "gif" format from: http://arxiv.org/ps/physics/9802024v1 
This figure "figure09.gif" is available in "gif" format from: http://arxiv.org/ps/physics/9802024v1 


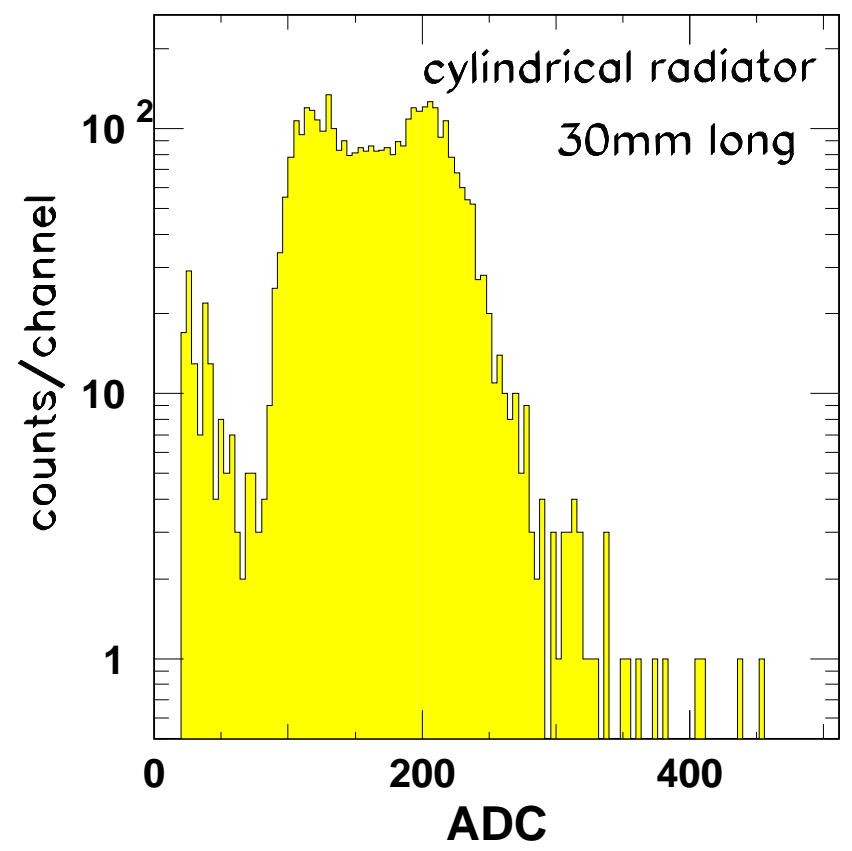



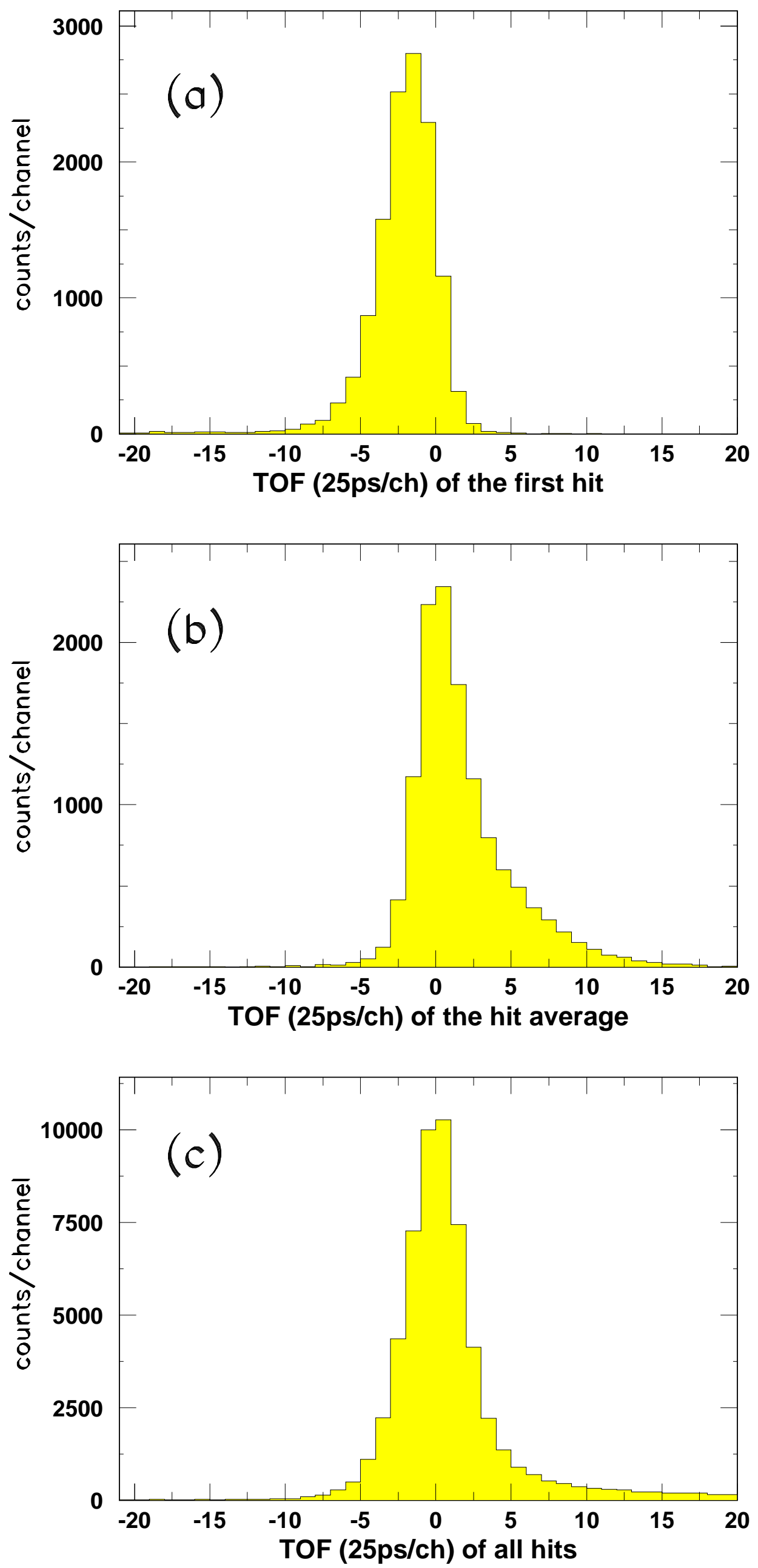
This figure "figure12.gif" is available in "gif" format from: http://arxiv.org/ps/physics/9802024v1 